\title{
On the Electromagnetic Fields Induced by Oceanic Internal Waves
}

\author{
Alan D. Chave \\ Institute of Geophysics and Planetary Physics, Scripps Institution of Oceanography, University of California at San Diego
}

\begin{abstract}
Model spectra for the electric and magnetic fields induced by oceanic internal waves are obtained by combining Green function solutions to the two electromagnetic modal equations with the Garrett-Munk kinematic description of the internal wave field. The poloidal magnetic mode is dominant at frequencies above $3 f$, where $f$ is the local Coriolis frequency, and self and mutual induction are not important over this range. The toroidal magnetic mode is increasingly important at frequencies below $3 f$ and is sensitive to the conductivity structure below the seafloor for nearinertial frequencies. The moored electric field is shown to be largely a measure of the local velocity field at high frequencies. The vertical electric field is sensitive to the horizontal velocity field, while the horizontal electric field primarily reflects the vertical velocity field and is quite small at the seaearth and sea-air interfaces. The magnetic field is a measure of the spatially averaged velocity field and is dominated by the poloidal magnetic mode. Electromagnetic boundary effects reduce the horizontal magnetic spectrum by decades at the seafloor and sea surface. At the seafloor and sea surface, internal wave-induced magnetic fields are within an order of magnitude of their externally induced counterparts, while in the ocean's interior, internal waves are probably the largest source of magnetic signals in the period range one day to one hour. The internal wave-induced electric field is not measurable except in the vertical component.
\end{abstract}

\section{INTRODUCTION}

Natural electromagnetic fields in the oceans are induced by both external ionospheric and magnetospheric electric current systems flowing far above the earth and by the dynamo interaction of ocean currents with the earth's magnetic field. The gross spatial and temporal morphology of the former are fairly well characterized at the earth's surface [e.g., Nishida, 1978], and external electromagnetic induction fields are frequently used in geophysical exploration, chiefly through the magnetotelluric method. By contrast, ocean-induced electromagnetic fields are less well understood, primarily due to a paucity of actual observations and the complexity of the ocean velocity field.

Studies of motional electromagnetic induction in the oceans are numerous, although the phenomena examined are confined principally to the barotropic tides [e.g., Larsen, 1968; Chave, 1983] and surface gravity waves [e.g., Weaver, 1965; Larsen, 1971]. In a recent study, Chave and Filloux [1984] showed that the seafloor electromagnetic power spectrum could be separated into nearly equal parts of ionospheric and oceanic origin over the period range 1 day to 1 hour and suggested a combination of a barotropic long wave and an internal wave mechanism to explain the result. Since internal waves are a ubiquitous feature of the oceans, a more detailed look at their electromagnetic effects is required to assess their role in the deep ocean electromagnetic environment. Previous attempts to compute internal wave-induced electromagnetic fields include Podney [1975] and Petersen and Poehls [1982], and the magnetic field gradients produced by intense, shallow water internal wave packets were observed by Podney and Sager [1979].

A unified picture of oceanic internal waves was pro-

Copyright 1984 by the American Geophysical Union.

Paper Number $4 \mathrm{C} 0893$.

0148-0227/84/004C-0893\$05.00 vided by the kinematic spectral description of Garrett and Munk [1972, 1975, see also Munk, 1981]; this development has inspired and guided much of the experimental effort of the last decade. The Garrett-Munk model utilizes linear theory and experimental data to predict displacement and velocity spectra of internal waves in both moored and towed configurations. Recent theoretical work has concentrated on the dynamics of internal waves and attempts to explain the universality in shape and amplitude of the observations. This research is reviewed by Munk [1981], Levine [1983], and Olbers [1983].

In this paper, theoretical spectra of the electromagnetic fields and their gradients generated by ambient internal waves are obtained by combining the poloidal and toroidal magnetic modal form of the field equations given by Chave [1983], which accounts for the influence of selfand mutual induction on the electromagnetic fields, with an eigenfunction expansion of the water velocity field and the Garrett-Munk variance spectrum. Both moored and towed sensors are considered. The spectral levels are shown to be quite high in the interior of the ocean, dwarfing any external contribution except during intense geomagnetic storms, but boundary effects reduce the spectra by orders of magnitude at the seafloor and sea surface, a phenomenon not noted by Petersen and Poehls [1982]. Magnetic gradient spectra are found to be near the limit of detection for moored instruments but are substantial in a towed sensor near the ocean's surface. These results indicate that internal wave contamination, along with surface gravity wave contributions, may be a source of noise in aeromagnetic surveying.

\section{HYDRODYNAMIC THEORY}

It is essential that the model for internal waves be consistent with the Garrett-Munk description to which the electromagnetic spectra will be tied; the linear form of the hydrodynamic equations used by Garrett and Munk [1972] will be followed. The $f$ plane equations under the Bous- 
sinesq approximation with the horizontal component of rotation, bottom topography, and wave current interactions neglected are

$$
\begin{aligned}
\partial_{t} u-f v & =-\partial_{x} p / \rho_{0} \\
\partial_{t} v+f u & =-\partial_{y} p / \rho_{0} \\
\partial_{t} w+N^{2} \zeta & =-\partial_{z} p / \rho_{0}
\end{aligned}
$$

where $u, v$, and $w$ are the east, north, and vertical fluid velocity components, $p$ is the pressure, $\rho_{0}$ is the mean fluid density, $\zeta$ is the particle displacement, $f$ is the Coriolis parameter, and $N$ is the buoyancy or BruntVäisälä frequency. Together with the incompressibility condition, these constitute a suitable set of equations for small-scale waves at moderate latitudes.

A single equation in $\zeta$ may be obtained from (1) and the incompressibility condition by eliminating $u, v, w$, and $p$, and vertical normal mode solutions are sought in the form

$$
\zeta(x, y, z)=\hat{\zeta}(z) e^{i(\vec{k} \cdot \vec{x}-\omega t)}
$$

where $\vec{k}=\eta \hat{x}+\xi \hat{y}$ is the horizontal wave number and $\omega$ is the angular frequency. The resulting eigenmode equation for the displacement is

$$
\partial_{z}^{2} \hat{\zeta}+\frac{N^{2}(z)-\omega^{2}}{\omega^{2}-f^{2}} k^{2} \hat{\zeta}=0
$$

Together with the usual boundary conditions of vanishing displacement at the seafloor $(z=-H)$ and sea surface $(z=0)$, (3) constitutes a Sturm-Liouville eigenvalue problem. The desired velocity components are obtained from (1):

$$
\begin{aligned}
u & =(\omega \eta+i f \xi) \partial_{z} \zeta / k^{2} \\
v & =(\omega \xi-i f \eta) \partial_{z} \zeta / k^{2} \\
w & =-i \omega \zeta
\end{aligned}
$$

Solutions of (3) can be obtained either approximately under the WKB formalism or analytically for specific types of buoyancy profiles. Garrett and Munk [1972] used analytical solutions associated with the exponential form

$$
N(z)=N_{0} e^{z / b}
$$

with $N_{0}=3 \mathrm{cph}$ and $b=1300 \mathrm{~m}$, which matches many deep ocean observations below the main thermocline, while Desaubies [1973] showed that other approximations introduced by Garrett and Munk effectively yielded a WKB form. For numerical purposes it is more convenient to obtain exact solutions of (3) for any $N(z)$ by converting it into an algebraic eigenvalue problem. Multiplying (3) by $\zeta$, integrating by parts, and using the boundary conditions yields

$$
\int_{-H}^{l} d z\left[\left(\partial_{z} \hat{\zeta}\right)^{2}-\frac{N^{2}(z)-\omega^{2}}{\omega^{2}-f^{2}} k^{2} \hat{\zeta}^{2}\right]=0
$$

to within a constant of integration. The term in brackets is the Lagrangian for the problem, and a stationary solution to (5) is sought. The displacement eigenfunctions will be parameterized in terms of a basis set $\left\{\phi_{j}\right\}$ such that

$$
\hat{\zeta}(z)=\sum_{j=1}^{M} a_{j} \phi_{j}(z)
$$

where the boundary conditions on $\hat{\zeta}$ are met by the $\left\{\phi_{1}\right\}$ and the number of terms $M$ is a free parameter. Appropriate choices for the basis functions include local types using spline functions or nonlocal types using orthogonal functions such as sinusoids; the former have better convergence properties but are usually more difficult to handle. For this problem, an appropriate choice is the set of eigenfunctions for the constant $N$ case, $\{\sin (j \pi z / H)\}$.

Substitution of (6) into (5) yields a quadratic form to be minimized in the usual way with respect to the expansion coefficients $a_{j}$. The result can be expressed as the algebraic eigenvalue problem

$$
\lambda^{2} \mathbf{A x}=\mathbf{B x}
$$

where $\lambda^{2}=k^{-2}$ is the eigenvalue, $\mathbf{x}=\left[a_{1}, \cdots, a_{M}\right]^{T}$ is the eigenvector, and $\mathbf{A}$ and $\mathrm{B}$ are $M \times M$ matrices with elements

$$
\begin{aligned}
A_{i j} & =\int_{-H}^{l} d z \partial_{z} \phi_{i}(z) \partial_{z} \phi_{j}(z) \\
B_{i j} & =\left[\int_{-H}^{l} d z\left(N^{2}(z)-\omega^{2}\right) \phi_{i}(z) \phi_{j}(z)\right] /\left(\omega^{2}-f^{2}\right)
\end{aligned}
$$

If the basis set is orthogonal, then $\mathbf{A}$ is diagonal and positive definite, while $\mathbf{B}$ is symmetric but is positive definite only if $\omega<N$ for all $z$. The problem (7) can then be transformed to standard form using a Cholesky decomposition of $\mathbf{A}$ and solved by standard methods using a package like EISPACK [Smith et al., 1976]. It is convenient to parameterize $N(z)$ in terms of splines, and the resulting matrix elements are computed quickly and accurately with the fast Fourier transform.

The eigenvectors obtained from (7) are orthonormal, and for consistency with Garrett and Munk [1972] each mode is scaled so that the vertically integrated energy is a constant

$$
\int_{-H}^{l} d z\left[u^{2}+v^{2}+w^{2}+N^{2} \zeta^{2}\right]=2 E
$$

where $E=N_{0}^{2} b^{3}$ for the exponential buoyancy profile. Figure 1 illustrates the displacement eigenfunctions for the first and third modes by using both an exponential buoyancy profile extending to the surface and the same form with a $500-\mathrm{m}$ mixed layer at the surface where $N=0$. Note the exponential decay of the displacement in the mixed layer and concomitant increase in the depth of maximum displacement. More complex density structures, including those given by experimental data, are no more difficult to handle.

\section{ELECTROMAgNETIC THEORY}

The framework for the theory of electromagnetic induction to be used in this paper was constructed by Chave [1983]. The Maxwell equations in the quasistatic limit, where the displacement current is neglected, govern the electromagnetic fields

$$
\begin{gathered}
\nabla \cdot \vec{B}=0 \\
\nabla \times \vec{E}+\partial_{\imath} \vec{B}=0
\end{gathered}
$$




$$
\nabla \times \vec{B}-\mu \sigma \vec{E}=\mu \mathrm{J}^{0}
$$

where the symbols have their usual meaning and $J^{0}$ is a specified source electric current density. Note that $\mu$ is the permeability of free space. For the study of controlled sources [e.g., Chave and Cox, 1982], $\vec{E}$ is the electric field outside of the finite extent source. For induction by moving seawater, the Lorentz transformations for the electromagnetic fields to terms of $O(v / c)$ yield

$$
\vec{J}^{0}=\sigma_{0}(\vec{v} \times \vec{F})
$$

where $\sigma_{0}$ is the conductivity of seawater, assumed constant, $\vec{v}$ is the water velocity field, and $\vec{F}$ is the static, sourceless geomagnetic induction. The electric field $E$ is the value for an observer fixed to the earth, while in a reference frame moving at velocity $\vec{v}$ the electric field is $\vec{E}^{\prime}=\vec{E}+\vec{\nabla} \times \vec{F}$ and the magnetic induction is unchanged.

The role of electric charge is often confused under the quasistatic approximation. Neglect of the displacement current in (11) is equivalent to filtering out fields with time scales of $O(\epsilon / \sigma)$, where $\epsilon$ is electrical permittivity, and requires that the total electric current be divergenceless. This implies that $\partial_{t} q=0$, where $q$ is electric charge, but Gauss's Law and (12) yield

$$
q=\epsilon[\nabla(l / \sigma) \cdot \vec{J}-(\nabla \times \vec{v}) \cdot \vec{F}]
$$

The quasistatic electric fields of these charges are large, and the part due to fluid vorticity is often dominant in the ocean. The electric currents which distribute these charges occur on a time scale of $O(\epsilon / \sigma)$ that is small compared to the quasistatic time scale, and they do not produce a significant time-varying magnetic field. An elaboration of this scaling as a singular perturbation problem is given by Backus [1982].

Solutions of (9)-(11) are obtained using potentials in a Cartesian coordinate system. By virtue of (9), the magnetic induction is completely specified by

$$
\vec{B}=\nabla \times(\Pi \hat{z})+\nabla_{h} \partial_{z} \Psi-\nabla_{h}^{2} \Psi \hat{z}
$$

where $\Pi$ and $\Psi$ are scalar functions which represent toroidal and poloidal magnetic (TM and PM) modes respectively. The source current in (11) may be written

$$
J^{0}=J_{z}^{0 \hat{z}}+\nabla_{h} T+\nabla \times(Y \hat{z})
$$

where $T$ and $Y$ are scalar functions which satisfy Poisson equations. For the source current in (12) the terms in (14) reduce to

$$
\begin{gathered}
J_{z}^{0}=\sigma_{0}\left(\vec{v}_{h} \times \vec{F}_{h}\right) \cdot \hat{z} \\
\nabla_{h}^{2} T=\sigma_{0}\left(\nabla_{h} \times \vec{v}_{h}\right) \cdot F_{z} \hat{z}+\sigma_{0}\left(\nabla_{h} \times v_{z} \hat{z}\right) \cdot \vec{F}_{h}
\end{gathered}
$$

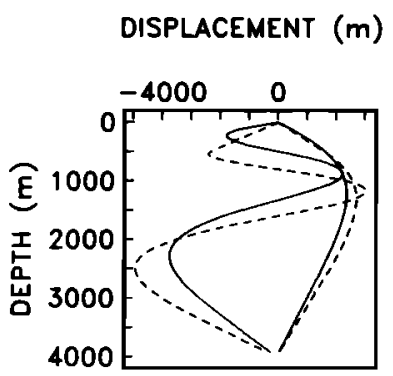

$.08 \mathrm{cph}$
DISPLACEMENT $(m)$

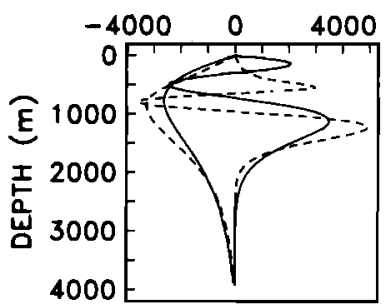

$2 \mathrm{cph}$
Fig. 1. The first and third mode displacement eigenfunctions at a latitude of $30^{\circ} \mathrm{N}$ and at near-inertial $(0.08 \mathrm{cph})$ and near-buoyancy (2 cph) frequencies. The solid lines correspond to the usual exponential buoyancy profile extending to the sea surface, while the dashed lines include a $500-\mathrm{m}$ mixed layer at the sea surface where the buoyancy frequency vanishes.

$$
\nabla_{h}^{2} Y=\sigma_{0}\left(\nabla_{h} \cdot \vec{v}_{h}\right) F_{z}-\sigma_{0}\left(\vec{F}_{h} \cdot \nabla_{h}\right) v_{z}
$$

where the horizontal and vertical velocity components have been separated in (16) and (17) due to their generally different scales in the ocean and spatial variations in $\vec{F}$ have been neglected.

If $\sigma$ is taken to vary only with the vertical coordinate, uncoupled differential equations for $\Pi$ and $\Psi$ may be derived from first principles

$$
\begin{gathered}
\nabla h \Pi+\sigma \partial_{z}\left(\partial_{z} \Pi / \sigma\right)-\mu \sigma \partial_{t} \Pi=-\mu J_{z}^{0}+\mu \sigma \partial_{z}(T / \sigma) \\
\nabla^{2} \Psi-\mu \sigma \partial_{t} \Psi=-\mu \Upsilon
\end{gathered}
$$

where the electric field is given by

$$
\begin{aligned}
E=-\left[\nabla h_{h}^{2} \Pi / \mu\right. & \left.+J_{z}^{0}\right] / \sigma \hat{z} \\
& +\nabla_{h}\left[\partial_{z} \Pi / \mu-T 1 / \sigma-\nabla \times\left(\partial_{t} \Psi \hat{z}\right)\right.
\end{aligned}
$$

Independence of the two modes is assured since the usual electromagnetic boundary conditions at horizontal interfaces are uncoupled.

\section{GREEN FUNCTIONS}

It is convenient to solve (18)-(19) by using the sinusoidal horizontal spatial and time dependence of (2). Green function solutions of the modal equations are useful because they explicitly incorporate all of the necessary boundary conditions for the problem and can be used to obtain solutions for arbitrary sources. Chave [1983] derived Green functions for an ocean of constant depth and constant conductivity, which may be written as

$$
\begin{gathered}
g_{\pi}\left(z, z^{\prime}\right)=-\frac{e^{-\beta\left|z-z^{\prime}\right|}}{2 \beta}-\frac{R_{L}^{\mathrm{TM}} e^{-2 \beta H} e^{-\beta\left(z+z^{\prime}\right)}-e^{\beta\left(z+z^{\prime}\right)}-R_{L}^{\mathrm{TM}}\left(e^{-2 \beta H} e^{\beta\left|z-z^{\prime}\right|}+e^{-\beta\left|z-z^{\prime}\right|}\right)}{2 \beta\left(1+R_{L}^{\mathrm{TM}} e^{-2 \beta H}\right)} \\
g_{\Psi}\left(z, z^{\prime}\right)=-\frac{e^{-\beta\left|z-z^{\prime}\right|}}{2 \beta}-\frac{R_{L}^{\mathrm{PM}} e^{-2 \beta H} e^{-\beta\left(z+z^{\prime}\right)}+R_{A}^{\mathrm{PM}} e^{\beta\left(z+z^{\prime}\right)}+R_{A}^{\mathrm{PM}} R_{L}^{\mathrm{PM}}\left(e^{-2 \beta H} e^{\beta\left|z-z^{\prime}\right|}+e^{-\beta\left|z-z^{\prime}\right|}\right)}{2 \beta\left(1-R_{A}^{\mathrm{PM}} R_{L}^{{ }^{\mathrm{PM}}} e^{-2 \beta H}\right)}
\end{gathered}
$$


where the solutions of (18) and (19) are given by

$$
\begin{gathered}
\hat{\Pi}=-\mu \int_{-H}^{\ell} d z^{\prime} g_{\pi}\left(z, z^{\prime}\right) \hat{J}_{z}^{0}\left(z^{\prime}\right)-\mu \int_{-H}^{\ell} d z \partial_{z^{\prime}} g_{\pi}\left(z, z^{\prime}\right) \hat{T}\left(z^{\prime}\right) \\
\hat{\Psi}=-\mu \int_{-H}^{\ell} d z^{\prime} g_{\Psi}\left(z, z^{\prime}\right) \hat{Y}\left(z^{\prime}\right)
\end{gathered}
$$

The electrical response of the conducting earth and insulating atmosphere are given by the reflection coefficients

$$
\begin{aligned}
& R_{L}^{\mathrm{TM}}=\left(\beta K / \sigma_{0}-1\right) /\left(\beta K / \sigma_{0}+1\right) \\
& R_{A}^{\mathrm{PM}}=(\beta-k) /(\beta+k) \\
& R_{L}^{\mathrm{PM}}=(\beta \Lambda / \mu-1) /(\beta \Lambda / \mu+1)
\end{aligned}
$$

where the TM and PM response functions $K$ and $\Lambda$ contain all of the information on the conducting earth necessary to compute them. Methods for their computation in terms of continued fractions for a layered earth are given by Chave [1983]. The effect of self induction is described by the induction parameter

$$
\beta=\sqrt{k^{2}-i \omega \mu \sigma_{0}}
$$

The first terms in (21) and (22) describe direct induction by the oceanic sources, while the complicated second terms contain interaction terms with the seafloor and surface; the denominators represent an infinite number of reflections between the ocean surface and bottom.

Self and mutual induction are not important for highfrequency internal waves, and (26) can be expanded in a first-order Taylor series for the limit $k^{2} \gg \omega \mu \sigma_{0}$

$$
\beta \equiv k(1-i \nu)
$$

where

$$
\nu=\omega \mu \sigma_{0} / 2 k^{2}
$$

is analogous to an induction or magnetic Reynolds number. For fundamental mode internal waves, $\nu$ varies from $10^{-1}$ to $10^{-4}$ as the frequency ranges from $2 f$ to $N$ at mid-latitudes, and the approximation breaks down for frequencies below $2 f$. It can be shown that the second term in (23) is $O(\nu)$ in this limit, and only the direct induction term need be considered. This is the case treated by Podney [1975] and adopted by Petersen and Poehls [1982]. The behavior of the TM mode is more complicated because of the different electromagnetic response function, but the TM mode is appreciable only for frequencies near the inertial, and the use of large wave number limits is not appropriate. Note that even the first term in (23) will yield a zone of width $k^{-1}$ at the seafloor and sea surface in which the field amplitude is reduced.

\section{The Electromagnetic FieldS}

A coordinate system oriented arbitrarily with respect to north is chosen for completeness. The source electric current terms in (15)-(17) are given by

$$
\begin{gathered}
J_{z}^{0}=\left[\left(\alpha_{y}+i \tau_{x}\right) \eta-\left(\alpha_{x}-i \tau_{y}\right) \xi\right] \partial_{z} \zeta / \mu \\
T=-\tau_{z} \partial_{z} \zeta / \mu+\left(\alpha_{y} \eta-\alpha_{x} \xi\right) \zeta / \mu
\end{gathered}
$$

$$
\Upsilon=-i \alpha_{z} \partial_{z} \zeta / \mu+\left(\alpha_{y} \xi+\alpha_{x} \eta\right) \zeta / \mu
$$

where

$$
\begin{aligned}
& \alpha_{x, y, z}=\mu \sigma_{0 \omega} F_{x, y, z} / k^{2} \\
& \tau_{x, y, z}=\mu \sigma_{0} f F_{x, y, z} / k^{2}
\end{aligned}
$$

are constants. The solutions to (18)-(19) given by (23)-(24) may be written

$$
\begin{aligned}
& \Pi=\tau_{2} \Gamma_{3}(z)-i\left(\tau_{x} \eta+\tau_{y} \xi\right) \Gamma_{4}(z) \\
& \Psi=i \alpha_{z} \Gamma_{1}(z)-\left(\alpha_{x} \eta+\alpha_{y} \xi\right) \Gamma_{2}(z)
\end{aligned}
$$

where the integrals are

$$
\begin{aligned}
& \Gamma_{1}(z)=\int_{-H}^{\ell} d z^{\prime} g_{\Psi}\left(z, z^{\prime}\right) \partial_{z^{\prime}} \hat{\zeta}\left(z^{\prime}\right) \\
& \Gamma_{2}(z)=\int_{-H}^{\ell} d z^{\prime} g_{\Psi}\left(z, z^{\prime}\right) \hat{\zeta}\left(z^{\prime}\right) \\
& \Gamma_{3}(z)=\int_{-H}^{\ell} d z^{\prime} \partial_{z^{\prime}} g_{\pi}\left(z, z^{\prime}\right) \partial_{z^{\prime}} \hat{\zeta}\left(z^{\prime}\right) \\
& \Gamma_{4}(z)=\int_{-H}^{\ell} d z^{\prime} g_{\pi}\left(z, z^{\prime}\right) \partial_{z^{\prime}} \hat{\zeta}\left(z^{\prime}\right)
\end{aligned}
$$

The electromagnetic fields follow from (13) and (20)

$$
\begin{aligned}
& E_{x}=-\omega \xi \Psi+i \eta\left(\partial_{z} \Pi / \mu-T\right) / \sigma_{0} \\
& E_{y}=\omega \eta \Psi+i \xi\left(\partial_{z} \Pi / \mu-T\right) / \sigma_{0} \\
& E_{z}=\left(k^{2} \Pi / \mu-J_{z}^{0}\right) / \sigma_{0} \\
& B_{x}=i \eta \partial_{z} \Psi+i \xi \Pi \\
& B_{y}=i \xi \partial_{z} \Psi-i \eta \Pi \\
& B_{z}=-k^{2} \Psi
\end{aligned}
$$

In practice the displacement and velocity eigenfunctions are computed numerically for a given frequency and mode number using (6)-(8), and the convolution integral terms in (30) are computed by standard quadrature methods to yield (28)-(29) and (31)-(36). There is an orientation dependence to $(31)-(36)$ due to a preferred direction for the geomagnetic field which shows up in the electromagnetic spectra as anisotropy.

\section{MOORED AND TOWED SPECTRA}

Following the notation of Garrett and Munk [1972], let $E(\eta, \xi, \omega)$ denote the energy spectral density per unit mass for the internal wave field at a fixed depth in the ocean. This quantity is a result of the complex and poorly understood dynamics of internal waves and has not been obtained from theory. The kinematic Garrett-Munk spectra, derived from observations, is horizontally isotropic, so that the two dimensional frequency-wave number spectrum is given by

$$
E(k, \omega)=2 \pi k E(\eta, \xi, \omega)
$$

and Munk [1981] gives an equivalent normal mode form where the wave number $k$ is replaced by the mode number $j$. 
Auto- and cross spectra or coherences of an observable quantity $\Phi$ are derived by normalizing the eigenfunctions from which $\Phi$ descends to unit energy using (8) and examining the moments of $\Phi$ projected along the frequency-wave number axes. The mean square value of complex $\Phi$ at a fixed depth is given by

$$
\left\langle\Phi^{2}\right\rangle=\int_{-\infty}^{\infty} \int^{\infty} d \eta d \xi \int_{0}^{\infty} d \omega \Phi(\eta, \xi, \omega) \Phi^{*}(\eta, \xi, \omega) E(\eta, \xi, \omega)
$$

where the asterisk denotes the complex conjugate. The power spectrum for a moored observer is the result of simultaneous contributions from all permissible wave numbers at a given frequency

$$
S_{\Phi \Phi}(\omega)=\int_{-\infty}^{\infty} \int^{\infty} d \eta d \xi \Phi(\eta, \xi, \omega) \Phi^{*}(\eta, \xi, \omega) E(\eta, \xi, \omega)
$$

Using the two-dimensional form of the energy density, (37) yields

$S_{\Phi \Phi}(\omega)=\frac{1}{2 \pi} \int_{0}^{\infty} d k E(k, \omega) \int_{0}^{2 \pi} d \theta \Phi(k, \theta, \omega) \Phi^{*}(k, \theta, \omega)$

where $(k, \theta)$ is the polar form of $(\eta, \xi)$. For numerical purposes, (39) is most easily computed by replacing the wave number integration by an equivalent summation over mode numbers.

A sensor which is towed at a velocity that is much larger than the internal wave phase velocity (less than $5 \mathrm{~m} / \mathrm{s}$ ) yields a projection of the wave number spectrum in the tow direction. Taking the $\hat{x}$ direction as the tow axis yields

$$
S_{\Phi \Phi}(\eta)=\int_{0}^{\infty} d \omega \int_{-\infty}^{\infty} d \xi\left[\Phi(\eta, \xi, \omega) \Phi^{*}(\eta, \xi, \omega) E(\eta, \xi, \omega)\right.
$$

$$
\left.+\Phi(-\eta, \xi, \omega) \Phi^{*}(-\eta, \xi, \omega) E(-\eta, \xi, \omega)\right]
$$

where equal contributions from positive and negative wave numbers in the tow direction are required. If the internal wave field is isotropic, there must also be equal contributions from positive and negative cross-tow wave numbers, reducing (40) to

$$
S_{\Phi \Phi}(\eta)=4 \int_{0}^{\infty} d \omega \int_{0}^{\infty} d \xi Z(\eta, \xi, \omega) E(\eta, \xi, \omega)
$$

where

$$
\begin{aligned}
Z(\eta, \xi, \omega)= & {\left[\Phi(\eta, \xi, \omega) \Phi^{*}(\eta, \xi, \omega)\right.} \\
& +\Phi(-\eta, \xi, \omega) \Phi^{*}(-\eta, \xi, \omega) \\
& +\Phi(\eta,-\xi, \omega) \Phi^{*}(\eta,-\xi, \omega) \\
& \left.+\Phi(-\eta,-\xi, \omega) \Phi^{*}(-\eta,-\xi, \omega)\right] / 4
\end{aligned}
$$

Substituting (37) and changing the variable of integration gives

$$
S_{\Phi \Phi}(\eta)=\frac{2}{\pi} \int_{0}^{\infty} d \omega \int_{\eta}^{\infty} d k Z(\eta, k, \omega) E(k, \omega) / \sqrt{k^{2}-\eta^{2}}
$$

For computational purposes, it is convenient to replace the wave number integration by the equivalent summation over mode numbers. The frequency integration is accomplished by numerical quadrature, while frequency and wave number are connected by the dispersion relation, and (42) is substantially more difficult to calculate than (39).

Internal wave electromagnetic spectra are obtained from (39) and (42) by substituting (31)-(36) or their gradients for $\Phi$. Computation of the azimuthal integration in (39) and (41) is rather tedious. For the moored spectra the coordinate system is fixed to the earth with the usual oceanographic convention, and since the earth's field is approximately geocentric, $F_{x}=0$ and $\tau_{x}=\alpha_{x}=0$ in (25)-(27). The azimuthal integrals have the canonical form

$$
\begin{aligned}
\int_{0}^{2 \pi} d \theta \Phi(k, \theta, \omega) \Phi^{*} & (k, \theta, \omega) \\
& =2 \pi A+\frac{3}{4} \pi B+\pi C+\frac{\pi}{4} D
\end{aligned}
$$

where $A-D$ are listed in Table 1 . The towed magnetic spectrum term in (41) has the standard form

$$
\begin{aligned}
Z(\eta, \xi, \omega)= & A A^{*} \eta^{4}+\left(B B^{*}+A C^{*}+A^{*} C\right) \eta^{2} \xi^{2} \\
& +C C^{*} \xi^{4}+D D^{*} \eta^{2}+E E^{*} \xi^{2}+F F^{*}
\end{aligned}
$$

where $A-F$ are contained in Table 2. The towed magnetic gradient form of (41) is even more complex and is omitted, but can be obtained by straightforward algebraic manipulation.

\section{Model SPECTRA AND Discussion}

Figures 2 and 3 show the moored electric and magnetic field vertical and horizontal components at various depths for a latitude of $30^{\circ}$, a seafloor conductivity of $0.005 \mathrm{~S} / \mathrm{m}$, and a total water depth of $4000 \mathrm{~m}$. The horizontal spectra are taken in the east direction, but (43) suggests that anisotropy between the north and east moored power spectra is at most a factor of two. The buoyancy profile is the usual exponential type given by Garrett and Munk [1972].

The moored electric field is primarily a measure of the local velocity and displacement fields, and the terms involving $\zeta$ and $\partial_{z} \zeta$ in Table 1 dominate the spectra except at low frequencies, where inductive effects are important. These quantities are associated with the TM mode, and the PM mode terms in Table 1 are small at all frequencies. The origin of these terms can be understood by considering a single mode propagating in the $\hat{x}$ direction and integrating the emf $\vec{v} \times \vec{F}$ in Ohm's Law for a moving medium in a closed loop located in the $x-z$ plane. Applying Stoke's Theorem, decomposing $\nabla \times \vec{v} \times \vec{F}$, and assuming constant $\vec{F}$ and fluid incompressibility yields

$$
\oint(\vec{v} \times \vec{F}) \cdot d l=\iint(\vec{F} \cdot \nabla) v_{y} d x d z
$$


TABLE 1. Moored Spectra Integrals

\begin{tabular}{|c|c|c|c|c|}
\hline Component & $A$ & $B$ & $C$ & $D$ \\
\hline $\int^{2 \pi} d \theta E_{x} E_{x}^{*}$ & - & $\left(a_{y} \Gamma_{2}\right)^{2}+\left(a_{y} \zeta / \gamma\right)^{2}$ & $\left(a_{2} \Gamma_{1}\right)^{2}+\left[b_{2} \partial_{2}\left(\Gamma_{3}+\zeta\right)\right]^{2}$ & $\left(b_{y} \partial_{z} \Gamma_{4}\right)^{2}+i a_{y}^{2} \zeta\left(\Gamma_{2}-\Gamma_{2}^{*}\right) / \gamma$ \\
\hline $\int^{2 \pi} d \theta E_{y} E_{y}^{*}$ & - & {$\left[b_{y} \partial_{2} \Gamma_{4}\right)^{2}$} & $\left(a_{2} \Gamma_{1}\right)^{2}+\left(b_{z} \partial_{2}\left(\Gamma_{3}+\zeta\right)\right]^{2}$ & {$\left[a_{y}\left(\Gamma_{2}+i \zeta / \gamma\right)\right]^{2}$} \\
\hline $\int d \theta E_{z} E_{z}^{*}$ & $\left(b_{2} k \Gamma_{3}\right)^{2}$ & - & $\left(a_{y} \partial_{z} \zeta / k \gamma\right)^{2}+\left[b_{y}\left(k^{2} \Gamma_{4}+\partial_{z} \zeta\right) / k\right]^{2}$ & - \\
\hline $\int_{0}^{2 \pi} d \theta B_{x} B_{x}^{*}$ & - & $\left(d_{y} \Gamma_{4}\right)^{2}$ & $\left(c_{z} \partial_{2} \Gamma_{1}\right)^{2}+\left(d_{2} \Gamma_{3}\right)^{2}$ & $\left(c_{y} \partial_{z} \Gamma_{2}\right)^{2}$ \\
\hline $\int_{0}^{2 \pi} d \theta B_{y} B_{y}^{*}$ & - & $\left(c_{y} \partial_{z} \Gamma_{2}\right)^{2}$ & $\left(c_{z} \partial_{z} \Gamma_{1}\right)^{2}+\left(d_{2} \Gamma_{3}\right)^{2}$ & $\left(d_{y} \Gamma_{4}\right)^{2}$ \\
\hline $\int_{0}^{2 \pi} d \theta B_{z} B_{z}{ }^{*}$ & $\left(c_{2} k \Gamma_{1}\right)^{2}$ & - & $\left(c_{y} k \Gamma_{2}\right)^{2}$ & - \\
\hline
\end{tabular}

The $\hat{y}$ component of fluid velocity for a wave propagating in the $\hat{x}$ direction is caused entirely by Coriolis deflection, so that the result is $O(f / \omega)$. At high frequencies, the net emf is very small, and the electric current it produces must be of the same order, so that $\vec{E} \cong-\vec{\nabla} \times \vec{F}$ at all save near-inertial frequencies in the vertical plane containing the propagation direction. In the notation of $(\mathbf{2 0 )}$, this means that $E \equiv-\nabla_{h} T / \sigma_{0}-J_{z} \% \sigma_{0} \hat{z}$, accounting for their dominance in Table 1 .

The gross behavior of the electric field spectra can be related directly to the Garrett-Munk spectra. The driving terms on the right side of (16) are in the ratio $f u: \omega w$, so that the second term is dominant at all save near-inertial frequencies, and the horizontal electric field is caused principally by the vertical velocity component. The vertical electric field is driven by the horizontal velocity field through (15). The Garrett-Munk model places the horizontal-to-vertical velocity spectra in the ratio

$$
\frac{S_{u u}}{S_{w w}}=\frac{N^{2}\left(\omega^{2}+f^{2}\right)}{\omega^{2}\left(\omega^{2}-f^{2}\right)}
$$

which ranges from a value near one at high frequencies to a very large number as the inertial frequency is approached, accounting for the much larger spectral level in the vertical electric field for frequencies below $N / 3$. The vertical velocity vanishes at the seafloor and sea surface, explaining the marked reduction in the horizontal electric spectra at those points, while at mid-depths the horizontal electric power level is both large and uniform due to the smoothing effect of summing many hydrodynamic modes. By contrast the vertical electric field spectra are quite red below the local buoyancy frequency for a fixed depth because the internal wave field shifts from predominantly vertical to predominantly horizontal motion as frequency decreases. The high frequency rolloff of the spectra shifts to lower frequencies as the observation depth increases, reflecting a decrease in the local Brunt-Väisälä frequency. Mutual and self induction are important for the TM mode at frequencies below $3 f$ at all depths, as the electrical wavelength of the induced fields becomes of order the depth of the ocean, requiring that electric current loops close within the earth rather than completely within the ocean.

The magnetic field is a measure of the spatially averaged velocity field, as can be seen in the presence only of convolution-type integral terms from (30) in Table 1 and as can be shown using the Biot-Savart law. The PM mode terms dominate the horizontal magnetic power spectra at all save the lowest frequencies because the PM electric current is large; the TM electric field is approximately the appropriate part of $-\vec{v} \times \vec{F}$, and the TM electric current is very small. The behavior of the spectra with depth can be understood by noting that the baroclinic internal wave velocity field results in horizontal electric current loops that alternate in sign with location in the water column. This results in reinforcement of the horizontal and near

TABLE 2. Coefficients for Towed Magnetic Spectra

\begin{tabular}{ccccccc}
\hline & $A$ & $B$ & $C$ & $D$ & $E$ & $F$ \\
\hline$B_{x}$ & $-i \alpha_{x} \partial_{z} \Gamma_{2}$ & $\tau_{x} \Gamma_{4}-i \alpha_{y} \partial_{z} \Gamma_{2}$ & $\tau_{y} \Gamma_{4}$ & $-\alpha_{z} \partial_{z} \Gamma_{1}$ & $i \tau_{z} \Gamma_{3}$ & - \\
$B_{y}$ & $-\tau_{x} \Gamma_{4}$ & $\tau_{y} \Gamma_{4}+i \alpha_{x} \partial_{z} \Gamma_{2}$ & $-i \alpha_{y} \partial_{z} \Gamma_{2}$ & $-i \tau_{z} \Gamma_{3}$ & $-\alpha_{z} \partial_{z} \Gamma_{1}$ & - \\
$B_{z}$ & - & - & - & $\alpha_{x} k^{2} \Gamma_{2}$ & $\alpha_{y} k^{2} \Gamma_{2}$ & $-i \alpha_{z} k^{2} \Gamma_{1}$ \\
\hline
\end{tabular}



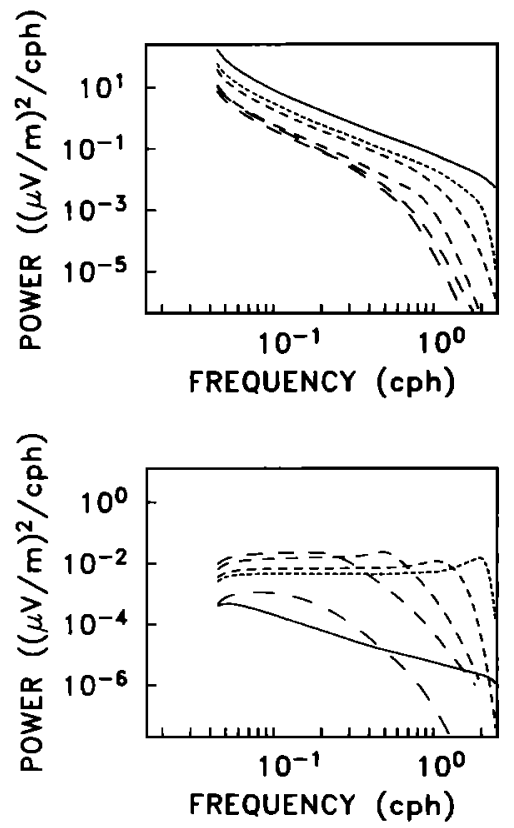

Fig. 2. Moored power spectra for (bottom panel) the north electric field and (top panel) vertical electric field at a latitude of $30^{\circ}$ in a 4000-m deep ocean, with a seafloor conductivity of $0.005 \mathrm{~s} / \mathrm{m}$ and for an exponential buoyancy profile consistent with the Garrett-Munk spectrum. The spectra are shown at the sea surface (solid line), and in order of increasing length (dashed line) at $500 \mathrm{~m}, 1000 \mathrm{~m}, 2000 \mathrm{~m}, 3000 \mathrm{~m}$, and the seafloor at $4000 \mathrm{~m}$.

cancellation of the vertical magnetic fields in the interior of the ocean and reduced horizontal fields at its boundaries. The uniformity of both vertical and horizontal interior spectra is a consequence of the spatial smoothing inherent in (30) and the presence of many hydrodynamic modes. Note that Petersen and Poehls [1982] missed the
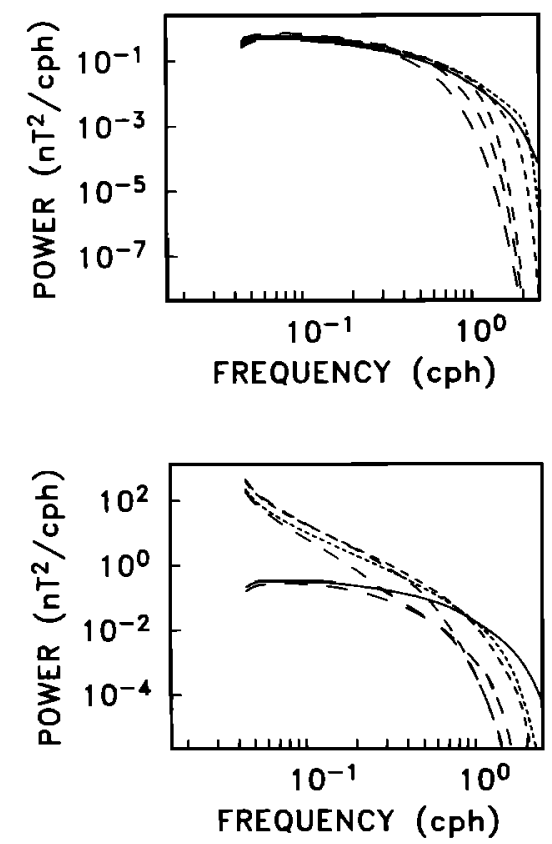

Fig. 3. Moored power spectra for (bottom panel) the north magnetic field and (top panel) the vertical magnetic field for the same parameters as in Figure 2.
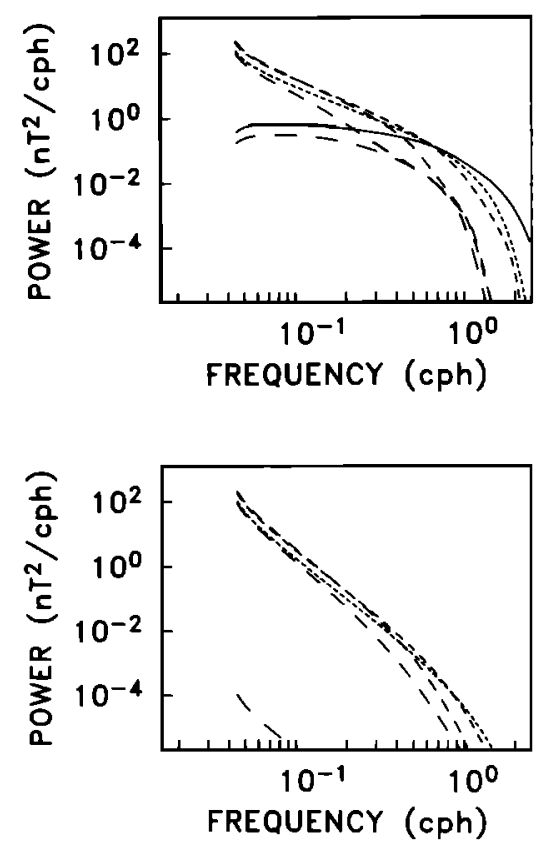

Fig. 4. The horizontal/components from Figure 3 separated into (top panel) PM mode and (bottom panel) TM mode components. The remaining parameters are as for Figure 2.

marked reduction in the horizontal magnetic spectrum near the sea surface; they used the electromagnetic formulation of Podney [1975], which neglects self and mutual induction and is not valid at low frequencies or near the seafloor.

Figure 4 shows the horizontal components of Figure 3 separated into PM and TM modes. The TM mode is important only close to the inertial frequency, and the TM mode horizontal magnetic field vanishes at the sea surface.

Figure 5 show model spectra of the total electric current normalized by the ocean conductivity $(J / \sigma=\vec{E}+\vec{v} \times \vec{F})$, as would be measured by a moored magnetic gradiometer set up to determine $\nabla \times \vec{B}$. This is very nearly the spectrum that would be obtained using towed electrodes, often called the GEK (geomagnetic electrokinetograph) field, with minor discrepancies occurring due to the use of (39) instead of (42). The horizontal current spectra are dominated by the PM mode part of $\vec{\nabla} \times \vec{F}$, and exceed their moored electric counterparts by $2-6$ decades at the seafloor and sea surface. At mid-depths, the horizontal current spectra are much larger than the horizontal electric spectra at the inertial end of the internal wave band, but the difference is small at near-buoyancy frequencies. Dropped electric field profilers [e.g., Sanford et al., 1978] use measurements of $\vec{J} / \sigma$ to infer $\vec{v}$, and the difference between the spectra of Figures 2 and 5 indicates the magnitude of the error caused by induced currents, suggesting small values at low frequencies but substantial ones at high frequencies. By contrast, there is almost no electric current flowing in the vertical direction, and the vertical current spectra of Figure 5 are substantially smaller than the vertical electric spectra of Figure 2. Note that the vertical current vanishes at the sea surface and is very small at the seafloor due to the large conductivity contrast between the ocean and earth. 

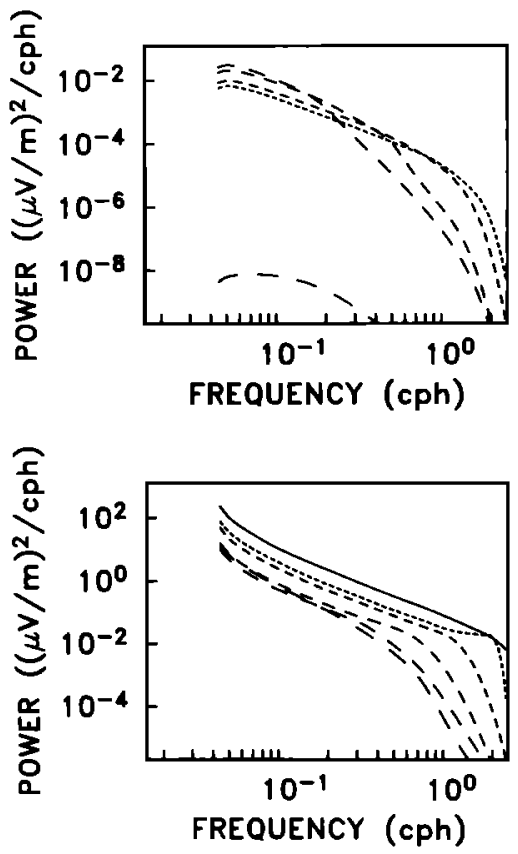

Fig. 5. Spectra of the total electric current per unit electrical conductivity in (bottom panel) the north direction and (top panel) the vertical direction for the same parameters as in Figure 2.

Internal waves exist with spatial scales to tens of kilometers, and penetration of their electromagnetic fields into the earth will occur to similar depths, depending on the subsurface conductive structure. The electrical structure of the suboceanic upper lithosphere can be divided into three regions: sediments, crust, and mantle. The electrical conductivity of unconsolidated sediment is close to that of seawater and decreases with lithification, but sediment cover is rarely more than a few hundred meters thick, and their effect on the electromagnetic fields is usually limited. The upper oceanic crust has a conductivity of $0.03-0.1 \mathrm{~S} / \mathrm{m}$, decreasing at a depth of $1.5 \mathrm{~km}$ by at least a decade [Becker et al., 1982], and the conductivity of the underlying mantle is probably even lower [Chave and Cox, 1982]. Chave and Cox [1984] obtained a mean conductivity of $0.001 \mathrm{~S} / \mathrm{m}$ for the upper oceanic lithosphere from continent-ocean boundary effects and argued that this is probably an overestimate away from mid-ocean ridges. The influence of mutual induction with the earth on low frequency oceanic electromagnetic fields has been emphasized by Cox [1981]. However, the effect of subsurface conductivity changes on internal wave-induced electromagnetic fields is small, reflecting the dominance of PM mode electric currents in the inductive process. An increase in the seafloor conductivity from $0.005 \mathrm{~S} / \mathrm{m}$ to $0.05 \mathrm{~S} / \mathrm{m}$ results in a $20 \%$ reduction in the horizontal electric spectra at near-inertial frequencies and a reduced effect as the frequency rises. By contrast, only a $4 \%$ increase in the horizontal magnetic spectra at low frequencies is observed, and negligible changes are seen in the vertical and current spectra.

Figure 6 shows the effect of replacing the uppermost ocean with a mixed layer, where the Brunt-Väisälä frequency vanishes and the hydrodynamic eigenfunctions decay exponentially. The horizontal electric and magnetic spectra are shown at depths of $250 \mathrm{~m}$ for a mixed layer of width 0,250 , and $500 \mathrm{~m}$ at $30^{\circ}$ latitude. The horizontal electric field power rises for the $250 \mathrm{~m}$ mixed layer, as the displacement peaks nearer the observation point (see Figure 1) and then decreases markedly as the mixed layer width increases and the peak displacement moves farther away. The horizontal magnetic field behaves in a similar fashion, although the size of the effect is smaller due to inherent spatial averaging, and the high frequency roll-off occurs at lower frequencies as the mixed layer depth increases. Note that the modes are normalized to the same integrated energy through (8) as the buoyancy structure is changed and, if inappropriate, would require some unknown level shifts between the spectra in the figure. Roth et al. [1981] argued that the Garrett-Munk spectrum is a lower limit or base state to the observed spectrum in the upper ocean, which supports the forms seen in the figure. Note that the magnetic field is affected by changes in the local hydrodynamic structure to a much lesser degree than either the electric field or a mechanical current meter would be, suggesting that a magnetometer could have some desirable features as an oceanographic instrument.

Figures 7 and 8 show examples of towed magnetic spectra below and above the sea surface as obtained from (42). The spectra decrease with wave number at a rate which increases as the wave number rises and is larger for the magnetic field than for the magnetic gradients. The towed spectrum above the ocean decreases more rapidly than that within the ocean as the wave number rises because the observation point to source point separation becomes large compared to the wavelength and high order modes are filtered out. Repeated tows at different heights above the ocean could in principle be used to separate the mode number dependence of the spectra.
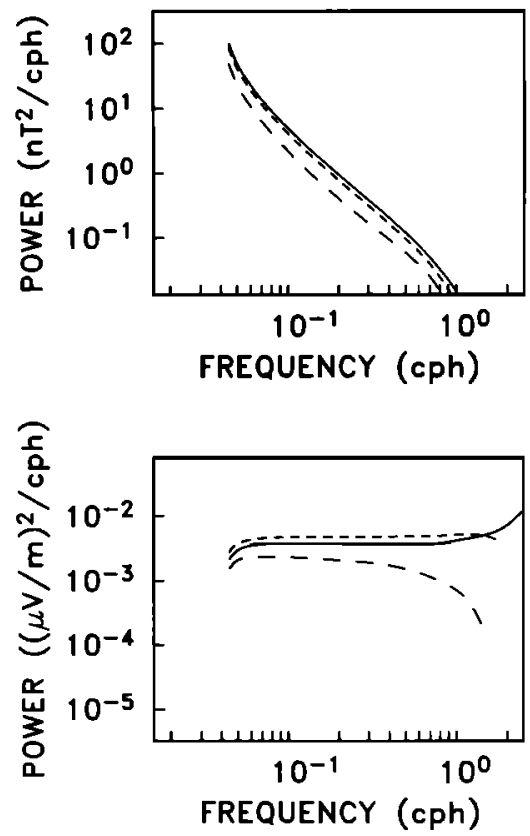

Fig. 6. The (bottom panel) horizontal electric and (top panel) magnetic field power spectra at $250 \mathrm{~m}$ for an exponential buoyancy profile extending to the sea surface (solid line), with a $250-\mathrm{m}$ surface mixed layer (short dashed line) and with a $500-\mathrm{m}$ surface mixed layer (long dashed line). See Figure 2 caption for details. 
It is important to compare the model spectra with those produced by other electromagnetic disturbances to estimate their relative importance. Chave and Filloux [1984] showed that the seafloor electromagnetic fields could be separated into nearly equal contributions from oceanic and ionospheric sources over the period range 1 day to 1 hour. The ionospheric part increases in a slow and predictable way as the sea surface is approached due to attenuation by the conducting ocean but does not change by over a decade even at high frequencies. The seafloor horizontal electric field oceanic part could not be explained by an internal wave mechanism, and even the increased levels seen in the ocean's interior in Figure 2 are not large enough to be significant. The seafloor horizontal magnetic field oceanic part was compatible with an internal wave source, and Figure 3 suggests that the internal wave magnetic fields will be dominant at mid depths, especially at low frequencies. By contrast, the vertical magnetic field ionospheric and oceanic parts are comparable at all depths, while the vertical electric field is entirely of oceanic origin.

The principal disturbances with similar spatial scale to internal waves to be compared with Figures 7 and 8 are surface wind waves and various ionospheric fields, especially micropulsations. Model spectra for wind waves can be obtained using (40), the electromagnetic field per unit wave height given by Chave [1983], and the directional wave variance spectrum [Phillips, 1977]. The latter may be written

$$
E(k, \theta, \omega)=F(k) g(\theta) \delta[\omega-\omega(k)]
$$

where the delta function in frequency indicates that a dispersion relation connects it with wave number. The wave number dependence is obtained by equating time
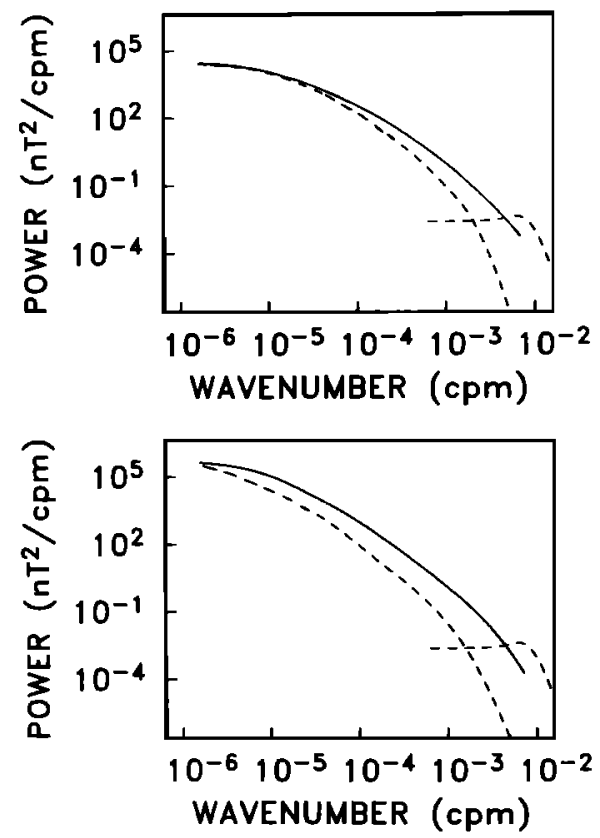

Fig. 7. Towed spectra of (bottom panel) the cross-track horizontal magnetic and (top panel) vertical magnetic fields at $100 \mathrm{~m}$ below (solid lines) and $50 \mathrm{~m}$ above (dashed line) the sea surface for the hydrodynamic model of Figure 2 . The short dashed line segment at the right of the figure is the contribution from surface wind waves in a $10 \mathrm{~m} / \mathrm{s}$ wind. See text for details.

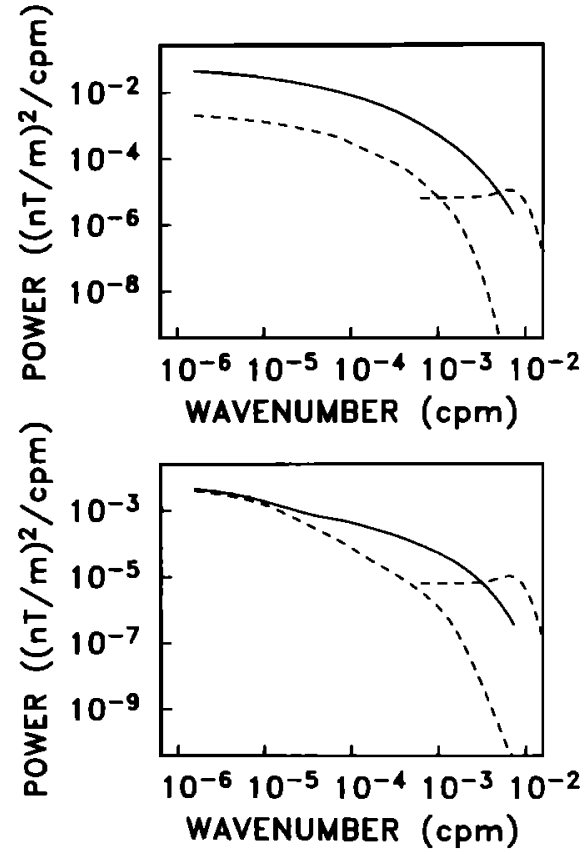

Fig. 8. Towed spectra for the cross-track gradient of (bottom panel) the cross-track magnetic field and (top panel) the vertical gradient of the vertical magnetic field at $100 \mathrm{~m}$ below (solid lines) and $50 \mathrm{~m}$ above (dashed line) the sea surface for the hydrodynamic model of Figure 2. The short dashed line segment at the right of the figure is the contribution from surface wind waves in a $10 \mathrm{~m} / \mathrm{s}$ wind. See text for details.

and space averages and using the Pierson-Moskowitz frequency spectrum to yield

$$
F(k)=\alpha e^{-\gamma\left(k / k_{m}\right)^{-2}} / 2 k^{4}
$$

where $\alpha$ is a dimensionless empirical constant in the range $0.001-0.01, \gamma$ is an empirical constant of $O(1)$, and $k_{m}$ is the wave number which corresponds to a wave phase velocity equal to that of the wind producing it. Tyler et al. [1974] used synthetic aperture radar scattering data to obtain the directional dependence

$$
g(\theta)=\Gamma\left(\frac{s}{2}+1\right) \cos ^{s}\left(\frac{\theta}{2}\right) / \sqrt{2 \pi} \Gamma\left(\frac{s+1}{2}\right)
$$

where $\Gamma$ is the gamma function, $s$ is an integer between 2 and 10 , and $\theta=0$ is the wind direction. Figures 7 and 8 include the results for a $10-\mathrm{m} / \mathrm{s} \quad(\sim 20-\mathrm{knot})$ wind, $\alpha=0.005$, and $s=2$ in an infinitely deep ocean. The spectra are dominated by the wind wave component at wave numbers above $5 \times 10^{-3} \mathrm{cpm}$, and any additional swell induced component could be large to $10^{-3} \mathrm{cpm}$. The slow fall off of the small wave number end of the model spectra is due to the very broad directional form implied by $s=2$; this results in very large cross-tow apparent wavelengths which induce large fields but are not realistic. It is probable that the internal wave spectra are dominant below $10^{-3} \mathrm{cpm}$, both at and above the sea surface.

Ionospheric current systems are broadband in form, and it is likely that external fields with wavelengths substantially smaller than the distance to the source will interfere destructively at the earth's surface. This argues for minimal ionospheric wave number effects above $10^{-5} \mathrm{cpm}$. Observations of the spatial coherence of ionospheric fields 
seem to be limited to Duffus et al. [1962] and Orange and Bostick [1965], who found high coherences over scales of $10-1000 \mathrm{~km}$ for source frequencies below $0.05 \mathrm{~Hz}$ and decreasing coherence at higher frequencies. Actual measurements of the wave number dependence of the magnetic gradients are practically nonexistent, although it is likely that spatial variations in the subsurface conductivity or susceptibility will dominate source effects. These variations are likely to be far smaller over the ocean than over land, but their actual size remains unknown. It is quite possible that internal wave-induced electromagnetic fields are an important source of noise in aeromagnetic surveying over the oceans.

It is interesting to compare the spectra of Figures 2-8 with the sensitivity of current generation instruments. The most sensitive magnetometers or gradiometers available use SQUID technology and have a sensitivity of $10^{-5} \mathrm{nT} / \sqrt{\mathrm{Hz}}$ [Clarke and Goldstein, 1981] or $10^{-5}(\mathrm{nT} / \mathrm{m}) / \sqrt{\mathrm{Hz}}$ (J. Clarke, private communication, 1983) at frequencies above $0.1 \mathrm{~Hz}$ and are degraded by $f^{-1}$ noise below this. These values can be converted to wave number space by equating the intrinsic frequency with the frequency of encounter $v k$, where $v$ is the tow velocity. For a tow at $100 \mathrm{~m} / \mathrm{s}$, appropriate for an aircraft, the noise level is $10^{-4} \mathrm{nT} / \sqrt{\mathrm{cpm}}$ or $10^{-4}(\mathrm{nT} / \mathrm{m}) / \sqrt{\mathrm{cpm}}$ above $10^{-3} \mathrm{cpm}$. Both the magnetic field and gradient spectra of Figures 7 and 8 are well above this level even if the degradation at small wave number is taken into account. The moored magnetic spectral values are measurable, and the electric field levels are marginally measurable in the oceans interior and well below the noise level at the seafloor, using the electrode noise and spectra given by Filloux [1973]. The vertical electric field spectra are easily measurable. There are serious problems in practice with moored magnetometers, since mooring motion produces a signal that is many decades larger than those that are of interest, and magnetic measurements must probably be confined to the seafloor. The sensitivity of electromagnetic devices to the velocity field is quite sizable; a signal level of $0.05 \mu \mathrm{V} / \mathrm{m}$ corresponds to a velocity of $10^{-3} \mathrm{~m} / \mathrm{s}$ at mid-latitudes, and such values could not be measured with mechanical current meters.

Acknowledgments. C. S. Cox, W. H. Munk, and D. S. Luther provided useful discussions on internal waves on many occasions. This work was sponsored by the Office of Naval Research under contract N00014-80-C-0440.

\section{REFERENCES}

Backus, G. E., The electric field produced in the mantle by the dynamo in the core, Phys. Earth Planet. Inter., 28, 191-214, 1982.

Becker, K., R. P. Von Herzen, T. J. G. Francis, R. N. Anderson, J. Honnorez, A. C. Anderson, J. C. Alt, R. Emmerman, P. O. Kempton, H. Kinoshita, C. Laverne, M. J. Mottl, and A. L. Newmark, In situ electrical resistivity and bulk porosity of the oceanic crust, Costa Rica Rift, Nature, 300, 594-598, 1982.

Chave, A. D., On the theory of electromagnetic induction in the earth by ocean currents, J. Geophys. Res., 88, 3531-3542, 1983 .

Chave, A. D., and C. S. Cox, Controlled electromagnetic sources for measuring electrical conductivity beneath the oceans, 1 , Forward problem and model study, J. Geophys. Res., 87, 5327-5338, 1982.
Chave, A. D., and C. S. Cox, Electromagnetic induction by ocean currents and the conductivity of the oceanic lithosphere, $J$. Geomagn. Geoelectr., in press, 1984.

Chave, A. D., and J. H. Filloux, Electromagnetic induction fields in the deep ocean off California: Oceanic and ionospheric sources, Geophys. J. R. Astron. Soc., 77, 143-117, 1984.

Clarke, J., and N. O. Goldstein, Magnetotelluric measurements, in Squid Applications to Geophysics, edited by $\mathrm{H}$. Weinstock and W. C. Overton, Jr., pp. 49-60, Society of Exploration Geophysicists, Tulsa, Okla., 1981.

Cox, C.S., On the electromagnetic conductivity of the oceanic lithosphere, Phys. Earth Plan. Inter., 25, 196-201, 1981.

Desaubies, Y. J. F., Internal waves near the turning point, Geophys. Fluid Dyn., 5, 143-154, 1973.

Duffus, H. J., J. A. Shaud, and C. S. Wright, Short range spatial coherence of geomagnetic micropulsations, Can. J. Phys., 40, 218-225, 1962.

Filloux, J. H., Techniques and instrumentation for study of natural electromagnetic induction at sea, Phys. Earth Planet. Inter., 7, 323-338, 1973.

Garrett, C. J. R., and W. H. Munk, Space-time scales of internal waves, Geophys. Fluid Dyn., 3, 225-264, 1972.

Garrett, C. J. R., and W. H. Munk, Space-time scales of internal waves: a progress report, J. Geophys. Res., 80, 291-297, 1975.

Larsen, J. C., Electric and magnetic field induced by deep sea tides, Geophys. J. R. Astron. Soc., 16, 47-70, 1968.

Larsen, J. C., The electromagnetic field of long and intermediate waves, J. Mar. Res., 29, 27-45, 1971.

Levine, M. H., Internal waves in the ocean: A review, Rev. Geophys. Space Phys., 21, 1206-1216, 1983.

Munk, W. H., Internal waves and small-scale processes, in Evolution of Physical Oceanography, edited by B. A. Warren and C. Wunsch, MIT Press, pp. 264-290, Cambridge, Mass., 1981.

Nishida, A., Geomagnetic Diagnosis of the Magnetosphere, SpringerVerlag, New York, 1978.

Olbers, D. J., Models of the oceanic internal wave field, Rev. Geophys. Space Phys., 21, 1567-1606, 1983.

Orange, A. S., and F. X. Bostick, Magnetotelluric micropulsations at widely separated stations, J. Geophys. Res., 70, 1407-1413, 1965.

Petersen, R. A., and K. A. Poehls, Model spectrum of magnetic induction caused by ambient internal waves, J. Geophys. Res., $87,433-440,1982$.

Phillips, O. M., The Dynamics of the Upper Ocean, 2nd, ed., Cambridge University Press, London, 1977.

Podney, W., Electromagnetic fields generated by ocean waves, $J$. Geophys. Res., 80, 2977-2990, 1975.

Podney, W., and R. Sager, Measurement of fluctuating magnetic gradients originating from oceanic internal waves, Science, 205, 1381-1382, 1979.

Roth, M. W., M. G. Briscoe, and C. H. McComas III, Internal waves in the upper ocean, J. Phys. Ocean, 11, 1234-1247, 1981.

Sanford, T. B., R. G. Drever, and J. H. Dunlap, A velocity profiler based on the principles of geomagnetic induction, Deep Sea Res., 25, 183-210, 1978.

Smith, B. T., J. M. Doyle, J. J. Dongarra, B. S. Garbow, Y. Ikebe, V. C. Klema, and C. M. Moler, Matrix Eigensystem Routines - EISPACK Guide, Springer-Verlag, New York, 1976.

Tyler, G. L., C. C. Teague, R. H. Stewart, A. M. Peterson, W. H. Munk, and J. W. Joy, Wave directional spectra from synthetic aperture observations of radio scatter, Deep Sea Res., 21, 989-1016, 1974.

Weaver, J. T., Magnetic variations associated with ocean waves and swell, J. Geophys. Res., 70, 1921-1929, 1965.

A. D. Chave, Institute of Geophysics and Planetary Physics, Scripps Institution of Oceanography, University of California at San Diego, La Jolla, CA 92093.

(Received March 16, 1984 accepted May 16, 1984.) 